\title{
Host-parasitoid spatial dynamics in heterogeneous landscapes
}

\author{
Alexandre H. Hirzel, Roger M. Nisbet and William W. Murdoch \\ A. H. Hirzel (Alexandre.Hirzel@unil.ch), Dept of Ecology and Evolution, Biophore Building, Univ. of Lausanne, CH-1015 \\ Lausanne, Switzerland. - R. M. Nisbet and W. W. Murdoch, Dept of Ecology, Evolution and Marine Biology, Univ. of California at \\ Santa Barbara, Santa Barbara, CA 93106-9610, USA.
}

\begin{abstract}
This paper explores the effect of spatial processes in a heterogeneous environment on the dynamics of a hostparasitoid interaction. The environment consists of a lattice of favourable (habitat) and hostile (matrix) hexagonal cells, whose spatial distribution is measured by habitat proportion and spatial autocorrelation (inverse of fragmentation). At each time step, a fixed fraction of both populations disperses to the adjacent cells where it reproduces following the Nicholson-Bailey model. Aspects of the dynamics analysed include extinction, stability, cycle period and amplitude, and the spatial patterns emerging from the dynamics.

We find that, depending primarily on the fraction of the host population that disperses in each generation and on the landscape geometry, five classes of spatio-temporal dynamics can be objectively distinguished: spatial chaos, spirals, metapopulation, mainland-island and spiral fragments. The first two are commonly found in theoretical studies of homogeneous landscapes. The other three are direct consequences of the heterogeneity and have strong similarities to dynamic patterns observed in real systems (e.g. extinction-recolonisation, source-sink, outbreaks, spreading waves).

We discuss the processes that generate these patterns and allow the system to persist. The importance of these results is threefold: first, our model merges into a same theoretical framework dynamics commonly observed in the field that are usually modelled independently. Second, these dynamics and patterns are explained by dispersal rate and common landscape statistics, thus linking in a practical way population ecology to landscape ecology. Third, we show that the landscape geometry has a qualitative effect on the length of the cycles and, in particular, we demonstrate how very long periods can be produced by spatial processes.
\end{abstract}

There is a large body of theory that describes the interactions of well mixed and spatially homogeneous "consumer" and "resource" populations (e.g. prey and predator; host and parasite or parasitoid). For an overview of this classical theory, see Murdoch et al. (2003). A second body of theory recognizes the importance of the distributions of populations in space (Murdoch et al. 2003 chapter 10); however, much of that work either assumes that habitat is either homogeneous or made up of identical patches. Real landscapes, of course, are heterogeneous. Their quality as habitat varies across space, and suitable habitat is commonly interspersed in a matrix of unsuitable habitat, which reduces the probability of successful dispersal. Here, we explore how this type of landscape structure impacts the finding from the previous, wellestablished, theory.
A key theme in the research that assumes perfect mixing or spatial homogeneity is identification of the interactions that lead to stable co-existence of consumer and resource or to particular patterns of fluctuation, such as cycles or chaos. An otherwise non-persistent system, described for example by the Nicholson-Bailey parasitoid-host model (Nicholson and Bailey 1935), may be stabilized via "self-organizing" processes, provided the system is large enough in relation to host and/or parasitoid dispersal to allow these processes to operate (Comins et al. 1992). One of three spatial patterns results, depending on the dispersal rates of the host and parasitoid: "crystal lattices", spiral patterns, and chaotic waves (Comins et al. 1992, Solé et al. 1992). Similar findings hold for continuous time and space (Sherratt et al. 2001). In systems with identical patches, population persistence of consumer resource 
systems can also be achieved through metapopulation dynamics - a balance between local extinction and recolonization (Maynard Smith 1974, Gurney and Nisbet 1978). There has been some limited study of patchy consumer-resource systems with non-identical patches (Murdoch et al. 1992), the most extreme situation being "mainland-island" or "source-sink" scenarios in which a smaller or less stable "sink" population is maintained by migrants from a single source (Pulliam 1988, Thomas and Kunin 1999).

In this paper, we show that by combining landscape structure and population dynamics into a common framework it is possible to generate all the above phenomena as particular outcomes from a single model. By varying habitat proportion, spatial auto-correlation and host dispersal rate, five important spatio-dynamical patterns can be identified: spatial chaos, spirals, metapopulation, mainland-island and fragmented-spirals. We describe the characteristics and the emergence conditions of these patterns and discuss the probability of finding them in nature.

From an application point of view, this study points to a few simple landscape and dynamic statistics that allow the conservation biologist to determine which processes are driving the system. Moreover, as the conditions for the emergence of the different patterns relate theoretical spatial dynamics to landscape statistics, they have the potential to synthesize insight from these two domains, thereby providing both the theoretician and the conservationist with novel ways of characterizing system behaviour.

\section{Model}

Consider a landscape invaded by populations of a host and its specialist parasitoid. Some parts of the landscape, henceforth designated "habitat," provide a suitable habitat for the host, where it can successfully reproduce and increase in density; in contrast, other parts, hence-forth designated "matrix," are largely unsuitable and colonizing populations decrease. Parasitoids attack the hosts independently of the habitat suitability. Both host and parasitoid can disperse a short distance before reproducing and dying.

\section{Population model}

We model this situation as follows: the landscape is represented by a hexagonal lattice of isometric cells - or hexes - small enough to assume that inside them: (1) host conditions are homogeneous, and (2) parasitism is random. The discrete-time dynamics on the landscape follow the spatial generalisation of the original Nicholson-Bailey (1935) model (from now on NB model) developed by Comins et al. (1992): the dynamics of each generation consist of a dispersal phase followed by a reproduction-and-parasitism phase.

During the dispersal phase, a fraction $\mu_{\mathrm{N}}$ of the hosts and $\mu_{\mathrm{P}}$ of the parasitoids leaves each hex to distribute themselves uniformly among the six neighbours, while the remainder stays in their original cell. For reasons discussed in the next section, the lattice has a torus topology, meaning that hexes located along a boundary are connected to the hexes of the opposite side. Accordingly, for each hex $\mathrm{i}$ the equations for the dispersal phase are:

$\mathrm{N}_{\mathrm{i}, \mathrm{t}}^{\prime}=\mathrm{N}_{\mathrm{i}, \mathrm{t}}-\mu_{\mathrm{N}} \mathrm{N}_{\mathrm{i}, \mathrm{t}}+\frac{1}{\mathrm{k}} \mu_{\mathrm{N}} \sum_{\mathrm{j} \text { in adj. hexes }} \mathrm{N}_{\mathrm{i}, \mathrm{t}}$

$\mathrm{P}_{\mathrm{i}, \mathrm{t}}^{\prime}=\mathrm{P}_{\mathrm{i}, \mathrm{t}}-\mu_{\mathrm{P}} \mathrm{P}_{\mathrm{i}, \mathrm{t}}+\frac{1}{\mathrm{k}} \mu_{\mathrm{N}} \sum_{\mathrm{j} \text { in adj. hexes }} \mathrm{P}_{\mathrm{i}, \mathrm{t}}$

where $\mathrm{N}_{\mathrm{i}, \mathrm{t}}$ and $\mathrm{P}_{\mathrm{i}, \mathrm{t}}$ are the host and parasitoid population densities in hex $\mathrm{i}$ at time $\mathrm{t}$, and $\mathrm{N}^{\prime}$ and $\mathrm{P}^{\prime}$ the corresponding densities after the dispersal. In the summation term, $j$ varies over the hexes adjacent to hex $\mathrm{i}$, and $\mathrm{k}$ is the number of neighbours, in this case, $\mathrm{k}=6$.

During the reproduction-and-parasitism phase, the population densities change according to the original NB model:

$\mathrm{N}_{\mathrm{i}, \mathrm{t}+1}=\lambda_{\mathrm{i}} \mathrm{N}_{\mathrm{i}, \mathrm{t}}^{\prime} \exp \left\{-\mathrm{aP}_{\mathrm{i}, \mathrm{t}}^{\prime}\right\}$

$\mathrm{P}_{\mathrm{i}, \mathrm{t}+1}=\mathrm{N}_{\mathrm{i}, \mathrm{t}}^{\prime} \mathrm{c}\left(1-\exp \left\{-\mathrm{aP}_{\mathrm{i}, \mathrm{t}}^{\prime}\right\}\right)$

where $\mathrm{a}$ is the per capita parasitoid attack rate, $\mathrm{c}$ is the conversion efficiency of parasitized hosts into adult parasitoids of the next generation, and $\lambda_{i}$ is the hexdependent host growth rate.

Following Comins et al. (1992), we assume that population densities are scaled so that a and $\mathrm{c}$ are set to one in all hexes. Thus variation in habitat quality across the landscape is assumed to be expressed solely through host growth rate, and the set of parameters $\left\{\lambda_{\mathrm{i}}\right\}$ represents the growth rate of the host population in each hex. Each hex is either habitat, with host growth rate $\lambda_{H}$, or unsuitable matrix, with host growth rate $\lambda_{M}$. Several growth rate values were explored but, unless stated otherwise, all results presented below were obtained using $\lambda_{\mathrm{H}}=2$ (in absence of parasitoid, the host density doubles at each generation.) and $\lambda_{\mathrm{M}}=0.01$ (rapid geometric decline with a small, but non-zero rate of host reproduction). Whenever host or parasitoid density drops below an arbitrary threshold of $10^{-10}$ in a hex, the local population is considered extinct and set to zero. There is no upper limit. Trial simulations showed that neither the precise value of the extinction threshold nor of an imposed maximal host density, if chosen within reasonable ranges, changed the outcome significantly. The NB model's simplicity makes it a 
perfect choice for investigating general mechanisms. In the discussion, we address how adding more complexity would alter our results.

\section{Landscape structure}

In this paper, a "landscape" is defined as the lattice of hexagonal cells characterized by the local host growth rate $\lambda_{\mathrm{i}}$, and a "patch" is defined as a set of one or more habitat hexes connected by at least one common side, and surrounded by matrix. Random landscapes can be generated by a wide range of algorithms (With 1997, With and King 1997, Andren 1999, Hill and Caswell 1999, Hiebeler 2000, Tischendorf 2001, Hargrove et al. 2002). We use the sequential Gaussian algorithm (Gomez-Hernandez and Srivastava 1990, Goovaerts 1998, Hirzel 2001), a method commonly used in geostatistics that is based on two parameters readily measurable in the field, namely habitat proportion, h, and spatial autocorrelation range of the landscape structure, $\alpha$.

First, a random landscape is generated using the sequential Gaussian algorithm, which we adapted to comply with the torus topology (Appendix 4). It assigns an interim random value $\mathrm{z}_{\mathrm{i}}$ to every hex, which is drawn from a Gaussian distribution with mean $=0$ and $S D=$ 1 , while controlling their spatial autocorrelation; the key parameter here is the autocorrelation range $\alpha$, defined as the distance (expressed in hex number) between hexes at which their $\mathrm{z}_{\mathrm{i}}$ have, on average, a correlation of 0.05 , assuming that correlation decreases geometrically with distance (Goovaerts 1998). Therefore, when the range is $\alpha=0$, the landscape is purely random and therefore "rough": knowing the $z_{i}$ value of a hex gives no indication to the value of an adjacent hex. Conversely, the larger the autocorrelation range, the smoother the landscape: the closer two hexes are, the smaller the expected difference is between their $z_{i}$ values. Second, we transform the continuous $z_{i}$ into binary $\lambda_{\mathrm{i}}$. We determine the proportion $\mathrm{h}$ of the hexes that are habitat, by computing a critical value of the parameter $\mathrm{z}$ using an inverse Gaussian distribution. Hexes whose z-value exceeds this critical value are habitat (growth rate $\lambda_{\mathrm{I}}=\lambda_{\mathrm{H}}$,) whilst the ones with values under the critical value are considered matrix $\left(\lambda_{I}=\lambda_{M}\right)$. Examples of landscapes generated this way are shown in Fig. 1.

The torus topology minimizes the potential effects of system size on dynamics; indeed, in such a topology, every hex or patch can be thought of as being at the centre of the landscape. Preliminary simulations convinced us that, consistently with the findings of Comins et al. (1992), the type of the boundary had no qualitative effect on the results.

\section{Simulation design}

The model defined above is controlled by four parameters, $\alpha$ and $\mathrm{h}$, which characterize the landscape, and the dispersal rates $\mu_{\mathrm{N}}$ and $\mu_{\mathrm{P}}$. We performed our simulations on a $30 \times 30$ lattice, for which we chose five values between 0 and 15 for the autocorrelation range $(\alpha)$ and five values between 0.1 and 0.9 for the habitat proportion (h). For each combination of these parameters, five random landscapes were generated. This process yielded 125 different landscapes.

These landscapes defined the environments within which different dispersal strategies were investigated by varying the dispersal rates $\mu_{\mathrm{N}}$ and $\mu_{\mathrm{P}}$ between 0.1 and 1. As initial condition, we assumed that all hexes in the landscape were empty, except for one hex, located in the centre of the biggest patch; its host and parasitoid densities were randomly and independently drawn from the range $[1,10]$. For each set of dispersal rates, ten simulations were run differing only in initial populations densities.

The simulations were allowed to run until: (1) the parasitoid went extinct; (2) the system attained a bounded fluctuations state (the standard deviation of the host density computed on the last 500 time steps was smaller than 1); or (3) 2000 generations elapsed, whichever came first. In most of the cases, however, either extinction or limit behaviour occurred before the 500th generation.

Finally, when the system was found to be persistent, the last 256 generations were used to compute several global statistics: mean and variance of the population densities and cycle amplitude, period and signal-tonoise ratio, assessed by a discrete Fourier analysis (Press et al. 1989).

Other experiments were made where we varied lattice size, dispersal neighbourhood radius, boundary conditions or patch/matrix growth rate. Their results are not discussed, unless they were qualitatively different from those produced by the above design.

\section{Results}

The outcome of the simulations was almost entirely determined by the system parameters, the only effect of the random initial densities being on the probability of early extinction. This probability drops when initial (scaled) host density is much higher (10:1) than (scaled) parasitoid density, and increases when this imbalance is reversed (few hosts, many parasitoids in one patch).

The most drastic effect of the parameters is on the persistence of the system. Extinctions (i.e. one species becoming extinct across the whole landscape) occur on two scales - local and landscape. The reason is the same in both cases: following oscillations of increasing 


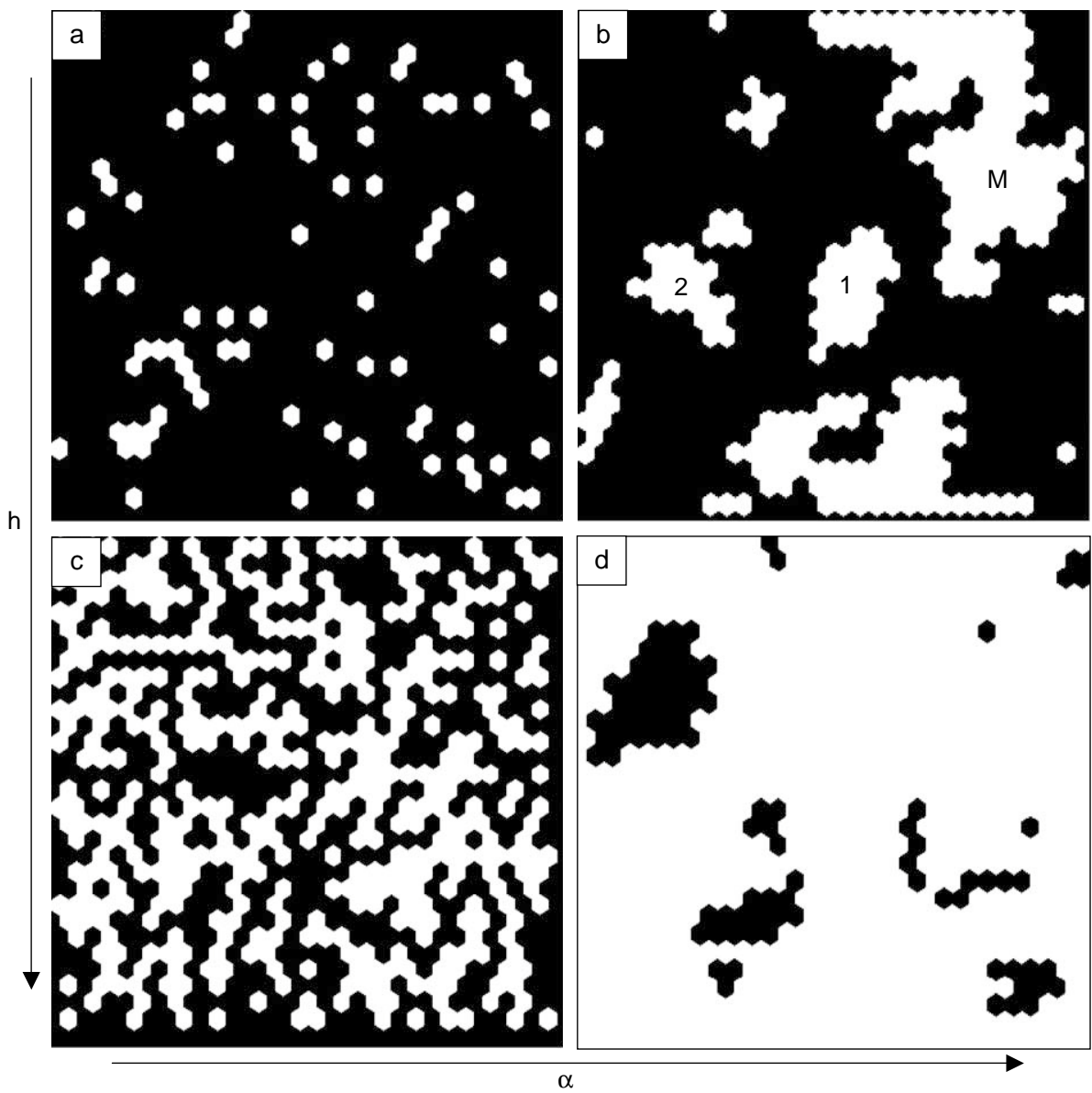

Fig. 1. Examples of the four landscape paradigms showing typical spatial distribution of suitable (white) and unsuitable (black) hexes: (a) spotted $(\alpha=0, \mathrm{~h}=0.1)$, (b) archipelago $(\alpha=15, \mathrm{~h}=0.3)$, (c) labyrinth $(\alpha=0, \mathrm{~h}=0.5)$, (d) Swiss cheese ( $\alpha=15$, $\mathrm{h}=$ 0.9 ). In panel (b), the capital letters identify the mainland (M), a close island (1) and a far island (2) (Fig. 4); because of the torus topology, the two "parts" of patch M are actually connected through the upper and lower edge of the lattice.

amplitude, the parasitoid overexploits the host then dies out. (1) In early extinctions (typically $<15$ generations) both species fail to spread from the initially occupied patch so the NB oscillations are short-lived. (2) Late extinctions occur after the whole landscape has been colonized (typically $>100$ generations in our simulations); the populations become more and more synchronised, until they behave like a single NB system. Synchronization may occur either as series of parasitoid waves travelling across the whole landscape leaving the host density lower and lower at each passage, or, less frequently, as full synchronisation of all hexes cycling together to extinction. Both early and late extinctions are favoured by high host mobility; early extinctions are more frequent in fragmented, habitat-scarce, landscape while late ones happen usually in more homogeneous and habitat-rich landscapes. However, in most cases the system was persistent, and we focus on these cases now.
The dynamics observed in simulations that attained a persistent state are strongly dependent on landscape geometry and dispersal rates. Six classes of spatial dynamics can be delineated: spirals, spiral fragments, mainland-island, spatial chaos, metapopulation and crystal lattice. Among them, the crystal lattice pattern is of very restricted practical use as it appears only when parasitoid dispersal rates are high $\left(\mu_{\mathrm{P}}>0.98\right)$ and host dispersal rates are low $\left(\mu_{\mathrm{N}}<0.05\right)$. It can be found in most kinds of landscapes, the only constraint being the presence of at least one patch that is large enough $(>30$ hexes) to house such a pattern, a condition unlikely to be filled when $\alpha<4$ and $h<0.15$ simultaneously. As it only occurs within such a narrow range of parameter values, the crystal lattice pattern will not be addressed further.

The remaining five patterns change gradually from one type to another as parameter values change. In order to describe their characteristics and conditions of 
emergence, we chose four exemplary landscape geometries, defined by their $\alpha$ and $h$ values, which we will refer to as "Swiss-cheese", "archipelago", "labyrinth" and "spotted" (Fig. 1 and 2, Table 1). Although each geometry blends into the next, often entailing hybrid dynamics, they are representative - and somewhat extreme - situations enabling one to isolate and understand the forces at play. The landscape geometry is, with host dispersal rate, the most important factor controlling the emergence the spatio-dynamical patterns. We explore now each geometry and describe the patterns along the way.

\section{Swiss-cheese geometry (high spatial correlation $(\alpha=15)$ and high habitat fraction $(h=0.9))$}

This landscape is nearly homogeneous and exhibits dynamics similar to the situation described by Comins et al. (1992): when the host dispersal rate is low the dynamics are characterised by chaotic short-lived travelling waves - the spatial chaos pattern - but when host dispersal rate is high, the waves are regularly organised in one or more spirals: the spiral pattern. The main difference from the homogeneous case is that the situation previously described as "hard-to-start spirals" (Comins et al. 1992), occurring for high $\mu_{\mathrm{N}}$ and low $\mu_{\mathrm{P}}$, is much less likely to occur here (Fig. 5d, noemergence area). Indeed, in a homogeneous landscape, concentric waves or other highly symmetrical patterns develop and grow, leading to the eventual extinction of

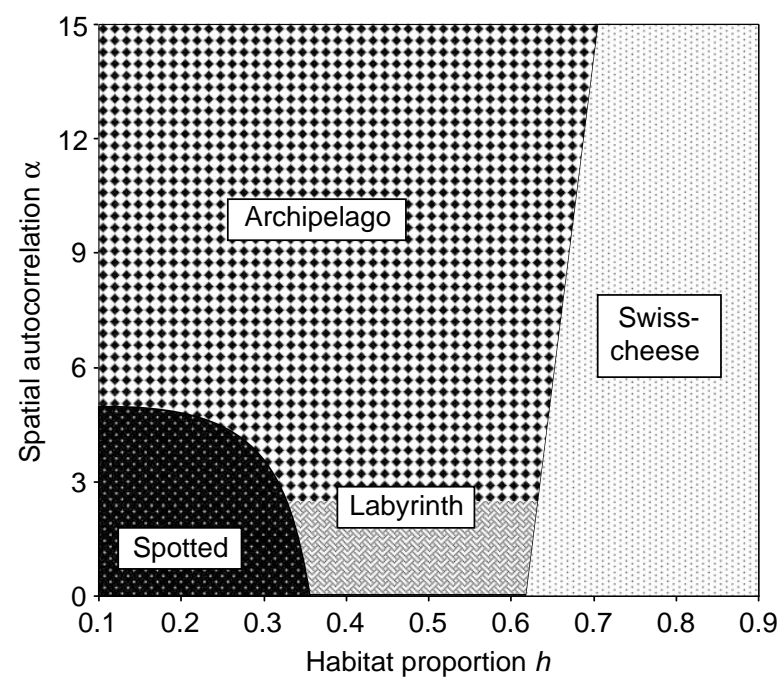

Fig. 2. Combination of habitat proportion $\mathrm{h}$ and spatial autocorrelation $\alpha$ defining the four landscape paradigms. The sharp boundaries between them are purely abstract as actually one type fades into the next. They were delineated on the base of four landscape statistics: Largest patch index, Mean patch size, Number of patches and Fractal dimension (Mcgarigal et al. 2002). the parasitoid. By contrast, the inhomogeneities introduced by the matrix holes in the Swiss-cheese landscape break the symmetry of the waves and make the selforganisation of spirals possible. Hosts can "hide" in these holes long enough to let the parasitoid wave move away before the parasitoid re-colonizes the depleted area left behind it. In these landscapes, the spiral vortices are often centred on or close to the holes.

\section{Archipelago geometry (high spatial correlation $(\alpha=15)$ and low habitat fraction $(h=0.3))$}

This landscape consists of a set of compact patches located at various distances from each other. This configuration produces the richest dynamic repertoire, with the outcome depending not only on dispersal rates but also on the particular geometry of the patches.

When host dispersal is sufficiently low (typically $\mu_{\mathrm{N}}<0.3$ ), the largest patches are big enough to develop chaotic patterns, allowing their populations to persist independently. As each patch can be considered an independent homogeneous landscape, the pattern here is essentially spatial chaos, in particular when all the patches are about the same size.

With higher host dispersal rates, the patches are too small to sustain a whole spiral pattern and only fragments, or travelling waves, can appear. These waves cross a patch leaving a depleted zone behind them; from there, the wave propagates to nearby patches and so on, eventually reaching all patches in the landscape. In most cases, however, the patches that were attacked first will be recolonised by dispersers coming from currently intact patches lying behind the wave, allowing the cycle to repeat itself ad infinitum, each patch experiencing quite regular extinctions and recolonisation (Fig. 4). The period of these cycles is dictated by the geometry of the islands through the constant-inflow dynamics described in Appendix 2. In some cases though, particularly when both host and parasitoid dispersal rates are high, this may lead to synchronisation of the patches and finally to global extinction. This process of wave propagation and recolonisation from the back is actually a spiral constrained by landscape boundaries. We call it the spiral fragment pattern.

However, this pattern is rarely present alone. In most cases, by chance, at least one of the patches is large enough to house small spiral arms, which entail more stable dynamics, smaller density variations and no extinction. This patch acts like a pool for the other smaller patches (for example, see Fig. $1 \mathrm{~b}$ in relation to the dynamics shown in Fig. 4). This leads to interesting and common dynamics where an archipelago of small patches experience recurrent extinctions, but are cyclically rescued by dispersers from the mainland. The detailed life-history of one of these islands is as follows: 
Table 1. Characteristics exhibited by the five spatio-dynamic patterns $(+=$ low,$++=$ medium, $+++=$ high $)$.

\begin{tabular}{|c|c|c|c|c|c|c|}
\hline \multirow[t]{2}{*}{ Pattern } & \multicolumn{2}{|c|}{ Equilibrium } & \multicolumn{2}{|c|}{ Cycles } & \multicolumn{2}{|c|}{ Commonness } \\
\hline & $\mathrm{N}^{*}$ & $\mathrm{~N} * / \mathrm{P}^{*}$ & Amplitude & Period ${ }^{*}$ & Noisiness & \\
\hline Spatial chaos & +++ & $\sim 2.5$ & + & $<10$ & +++ & +++ \\
\hline Metapopulation & ++ & $\sim 14$ & +++ & $<10$ & +++ & \\
\hline Mainland-island & + & $\sim 5$ & +++ & $>20$ & ++ & +++ \\
\hline Spiral fragments & + & $\sim 4$ & ++ & $15-20$ & ++ & +++ \\
\hline Spirals & +++ & $\sim 2$ & + & 11 & + & ++ \\
\hline
\end{tabular}

Number of generations. The absolute value of these figures depends on model specificities.

* Noise-to-signal ratio of a discrete Fourier analysis.

initially, the island is empty, but eventually it is colonised by hosts dispersing from the mainland. In the matrix, the host density is initially too low to enable the parasitoid to survive and thus to reach the island. Thus, in absence of external regulation, the insular host population begins to grow exponentially, producing an ever increasing overflow of dispersers. Eventually, this overflow, in combination with its mainland counterpart, generates a high enough host density in the matrix - the host bridge - to allow parasitoids to cross it. Once they colonise the island, they rapidly parasitize the huge host population, then go extinct themselves. The cycle can then start again with a new outbreak. For reasons developed in Appendix 3, the further away the island is from the mainland, the longer the time between two parasitoid invasions is (Fig. 1b, Fig. 4).

In our simulations, resulting periods commonly range from 30 to 80 generations, although much longer periods are not infrequent. Actually, within this model, any period length could theoretically be achieved providing the island is far enough from the mainland. However, in a more realistic framework, density-dependence in the host insular population would limit the length of the host bridge. We call it the mainland-island pattern. However, in spite of this terminology, the matrix lying between the mainland and the islands is not completely hostile territory: both species can reproduce there and the crossing often takes several generations.

\section{Spotted geometry (no spatial correlation $(\alpha=0)$ and very low habitat fraction $(h=0.1))$}

We jump now to the other end of the landscape spectrum to introduce the last pattern. In "spotted" landscapes, habitat is sparse and randomly distributed. The risk of rapid extinction is high (at best $60 \%$ but most of the time approaching 100\%). The few systems that manage to persist all have $\mu_{\mathrm{N}}<0.5$ (Fig. 5a) and experience wild oscillations over several orders of magnitude (Fig. 3b). Two effects combine to prevent the system from persisting: (1) most patches are composed of only one or two hexes and are surrounded by hostile habitat. They are thus smaller than the critical size needed to support a viable host population in the face of diffusion, unless the host dispersal rate is very low (Appendix 1). (2) Even when hexes are aggregated compactly enough that some exceed the critical size for host population growth, these patches are usually too small to house stabilising spatial patterns (including crystal lattice), and are on average too far apart to be able to rescue each other. Therefore, the only persistent dynamics appear in landscapes composed of a large number of small patches close to one another, combined with low host dispersal; in this configuration, the patches continuously and persistently experience extinction and recolonisation events, which, although the underlying processes are completely deterministic, are chaotic enough to look random; patch occupancy is much higher for the host than for the parasitoid because a substantial proportion of the host population is actually running through unsuitable areas. We call this spatio-dynamical pattern metapopulation.

\section{Labyrinth geometry (no spatial correlation $(\alpha=0)$ and medium habitat fraction $(h=0.5))$}

Starting from a spotted landscape and increasing the habitat proportion by adding habitat hexes randomly (keeping $\alpha=0$ ), initially, the mean patch size does not change noticeably but their number increases linearly, so patches become closer to each other. Between $h=0.4$ and $\mathrm{h}=0.6$, patch connection begins to increase and the actual number of patches drops rapidly as the percolation threshold (the value of $h$ at which, on average, all hexes are connected into only one patch, about 0.7 (Dussert et al. 1989)) is approached. In this range, the patches are highly interconnected, but have complex shapes with a low area/perimeter ratio (Fig. $1 b)$. This geometry is therefore an intermediate between the previously discussed landscapes and this results in a somewhat hybrid dynamics. In this kind of landscape, the diffusion effect (Appendix 1) generates asymmetrical influences on the host and the parasitoid: when host dispersal rate is low, most patches are larger than the critical size and the host colonises all suitable hexes, closely followed by the parasitoid, producing a spatial 

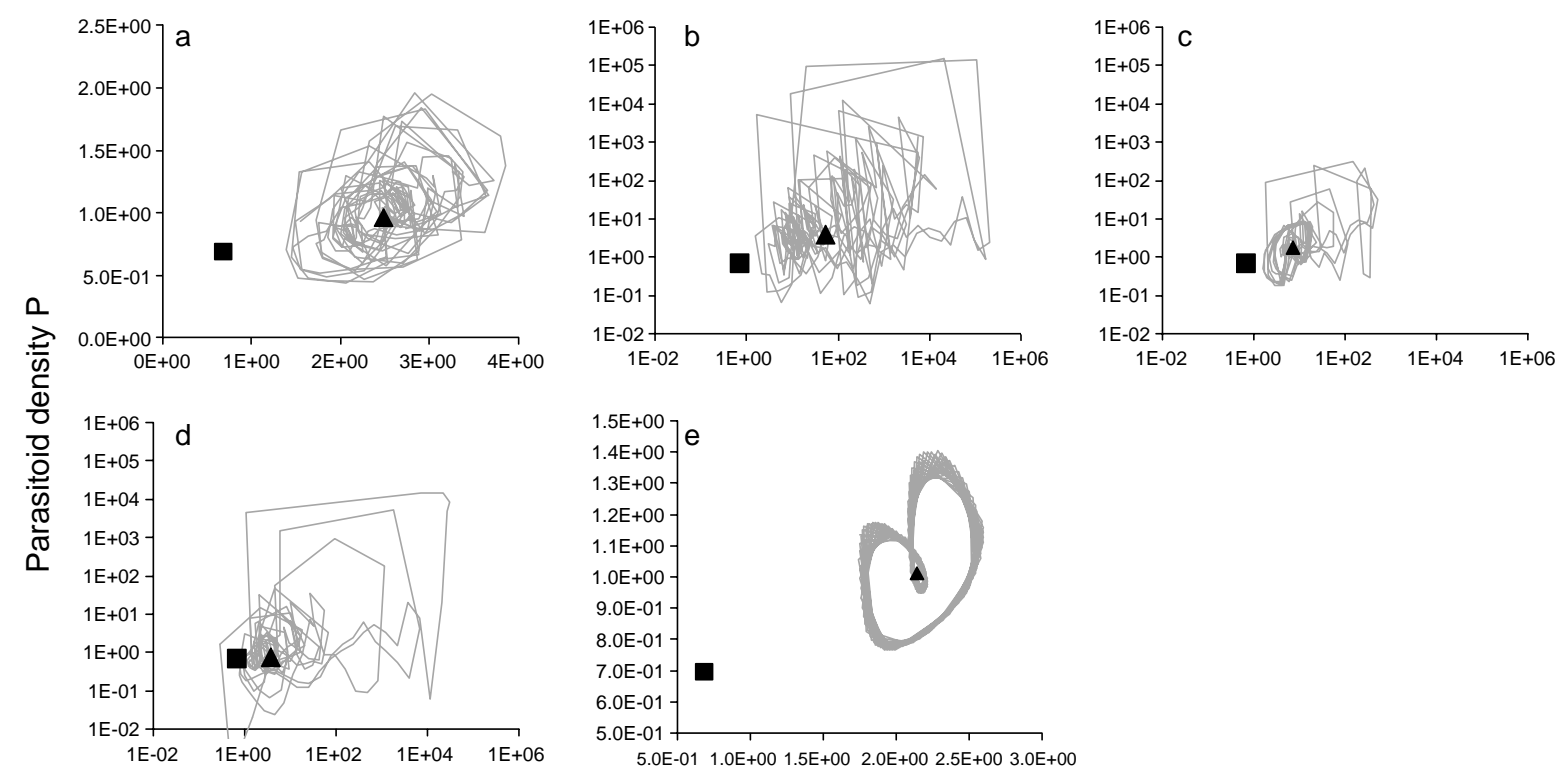

Host density $\mathrm{N}$

Fig. 3. Typical dynamics of the five patterns in the species space. (a) Chaos pattern $\left(h=0.5, \alpha=0, \mu_{N}=0.2, \mu_{P}=0.2\right)$ exhibits typical pseudo-random patterns due to the low host dispersal rate entailing a short range spatial correlation in the dynamics. (b) Metapopulation pattern $\left(h=0.1, \alpha=0, \mu_{N}=0.1, \mu_{P}=0.3\right)$ is driven by the same processes but in a much scarcer landscape, generating a much higher variance in the population densities (note the logarithmic scale). (c) In the spiral-fragment pattern $\left(\mathrm{h}=0.3, \alpha=15, \mu_{\mathrm{N}}=0.9, \mu_{\mathrm{P}}=0.1\right)$, higher dispersal rate in the host generates longer range and more regular spatial patterns; note the spiral-like pattern. (d) The mainland-island pattern $\left(h=0.3, \alpha=15, \mu_{N}=0.5, \mu_{P}=0.5\right)$ is a combination of two processes: the mainland is experiencing a chaotic dynamics while islands are submitted to recurrent outbreaks. e: The spiral pattern $\left(h=0.9, \alpha=15, \mu_{N}=0.9, \mu_{P}=0.5\right)$ occurs in more homogeneous landscapes when host dispersal is high, generating long range and very regular patterns. Note that spiral and chaos graphs have linear scale. The median densities (triangle) and Nicholson and Bailey's theoretical unstable equilibrium (square) are also shown. These numbers show the mean hex densities, taking into account only the suitable hexes.

chaos pattern. However, when host dispersal rate is high, the diffusion effect prevents the host from thriving in those areas where the patch shape is long and narrow (hexes connected to less than three neighbours, Appendix 1); although hosts can survive in these areas at low density, they are functionally closed to parasitoids. Therefore, the landscape as experienced by the parasitoids is actually much more fragmented than that experienced by its host and is indeed closer to archipelago geometry, generating spiral fragments or mainland-island patterns. Therefore depending on the host dispersal rate the functional landscape for the parasitoid will be either Swiss cheese or archipelago. This distinction between a functional and a physical landscape is potentially very important but, is beyond the scope of the present paper.

\section{Sensitivity analysis}

Qualitatively, the above results are insensitive to changes in parameters other than the four discussed above.
Varying matrix host growth rate $\left(\lambda_{M}\right)$ (while keeping $\lambda_{\mathrm{H}}=2$ ) has only small effects, increasing or decreasing patch isolation and, as a consequence, influencing early extinction risk. Reducing $\lambda_{M}$ to zero actually traps the system in the initial patch, thereby mimicking the homogeneous case investigated by Comins et al. (1992). Increasing $\lambda_{M}$ has no strong effect until it is close to a zero-growth matrix, $\lambda_{\mathrm{M}}=1$. The main result is then to decrease drastically or even completely remove the risk of early extinction in all cases except the Swiss-cheese landscapes where early extinction is always unlikely. The greatest change happens in the spotted landscapes where extinctions now occur almost only at $\mu_{\mathrm{N}}>0.7$. The zero-growth matrix eliminates the over-diffusion effect, so there is no critical patch size required for a viable host population. The late extinctions however, caused by global synchronisation, can still happen and their likelihood may be increased. A secondary effect is that the weaker spatial heterogeneity is no longer able to break the parasitoid wave front, producing dynamics more similar to those of a homogeneous landscape, particularly when the gaps between patches are small, as in the labyrinth and Swiss 


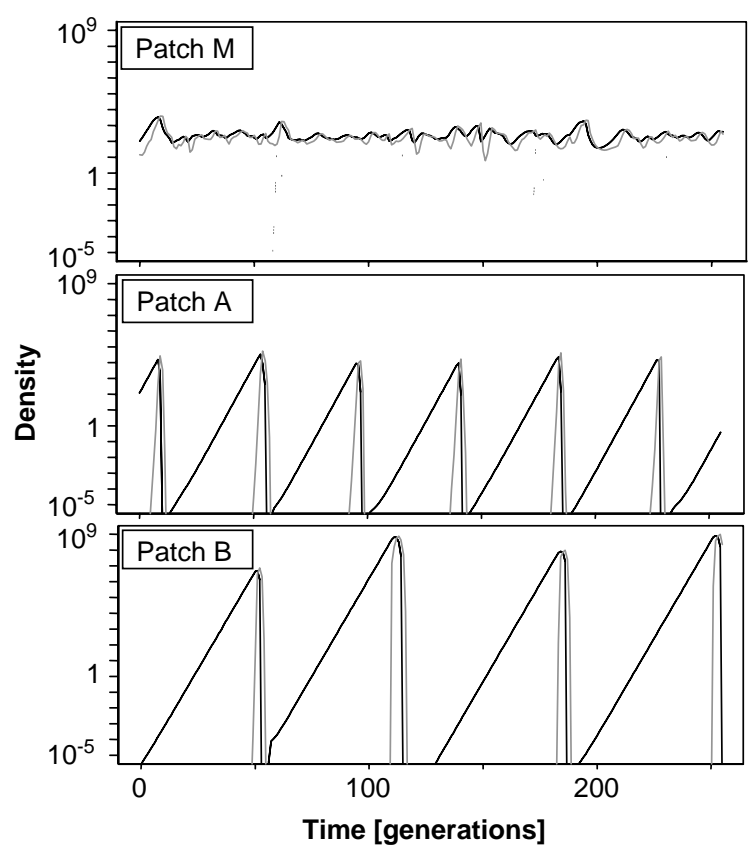

Fig. 4. Typical time-series following a "Mainland-Island" pattern showing the densities of the host (black) and parasitoid (grey) populations in three patches. The mainland (M) follows a sub chaotic dynamics and acts as a source for the close (A) and far (B) islands whose cycle period and amplitude increases with distance, due to the host-bridge effect (cf. Appendix 3). This simulation was done on landscape b) of Fig. 1 with $\mu_{\mathrm{N}}=\mu_{\mathrm{P}}=0.5$.

cheese geometries. In the latter, the discontinuities in the landscape can no longer favour spiral emergence by breaking wave front symmetry; accordingly, Swisscheese landscapes no longer show a significantly different behaviour from the homogeneous case. A third effect is to make the matrix a refuge for the host: on average, the host density is higher in the matrix than in the patches, which have a higher level of parasitoids. Finally, the host bridge between patches being permanent, the mainland-island pattern cannot emerge.

We also tested the effect of increasing growth rate $\left(\lambda_{\mathrm{H}}\right)$ in habitat while keeping $\lambda_{\mathrm{M}}=0.01$. Again, the main effect is to decrease early extinction risk although in a less drastic way. In spotted landscapes, the metapopulation pattern can now emerge for any system with $\mu_{\mathrm{N}} \leq 0.7$. By contrast, the late extinction risk is noticeably increased, particularly for the most homogeneous landscapes.

In the above simulations, dispersal range, i.e. the number of hexes dispersers can cross in one generation, was set to 1 . We tested the effect of increasing this value to 2 and 3 and, that was found to have little effect. Decreasing patch isolation somewhat reduced early extinction risk in spotted landscape, enabling a metapopulation pattern to emerge at $\mu_{\mathrm{N}} \leq 0.5$ and $\mu_{\mathrm{P}} \leq 0.8$. Longer dispersal range tended to shift chaotic dynamics toward more spiral-like patterns, but the spirals themselves have a longer wavelength, which made extinction more likely as they need larger homogeneous patches to persist. This tended therefore to shift to the left the emergence patterns shown in Fig. $5 \mathrm{~b}-\mathrm{d}$. It also shortened the length of the transient dynamics needed to achieve limiting behaviour. However, increasing the dispersal range entailed a much longer simulation time and it was unpractical to investigate longer ranges, at which the landscape could eventually become so well mixed it would behave like a non-spatial system.

\section{Summary of pattern characteristics}

Our results have led to the identification of five important spatio-dynamic patterns whose occurrence can be deterministically predicted on the base of landscape geometry and host dispersal rates. These patterns can be easily discriminated from each other by looking at their spatial dynamics, at their global dynamics in the phase space (Fig. 3) and at the global equilibrium statistics (mean host density, mean host/ parasitoid ratio, cycle amplitude, period and signal-tonoise ratio, see Table 1). The spatial chaos pattern (Fig. $3 \mathrm{a})$ is the most common as it emerges whenever the host dispersal is low in all but spotted landscapes, in which case the metapopulation pattern occurs. When host dispersal is medium or high, landscape geometry becomes a decisive factor. Swiss-cheese landscapes entail the emergence of the spiral pattern (Fig. 3e), labyrinth landscapes favour the spiral fragment pattern (Fig. 3c), whilst archipelago geometry generates chiefly mainlandisland pattern (Fig. 3d). These relationships between landscape geometry, host dispersal rates and the patterns are summarised in Table 2.

\section{Relating pattern to process}

It is possible to better understand the relationship between landscape and population dynamics by considering more mechanistically the processes that produce the patterns discussed in the previous section.

The NB model has fundamentally unstable dynamics and the only way to achieve population persistence is to allow the host to escape the parasitoid. However, the host must also be able to "reseed" the depleted areas. The mechanism for reseeding depends on the host dispersal rate. If the host has a low dispersal rate, synchronisation acts at a very short range. This allows reseeding points to occur close together. In a homogeneous context, this process generates spatial chaos, i.e. short-lived omni-directional travelling 

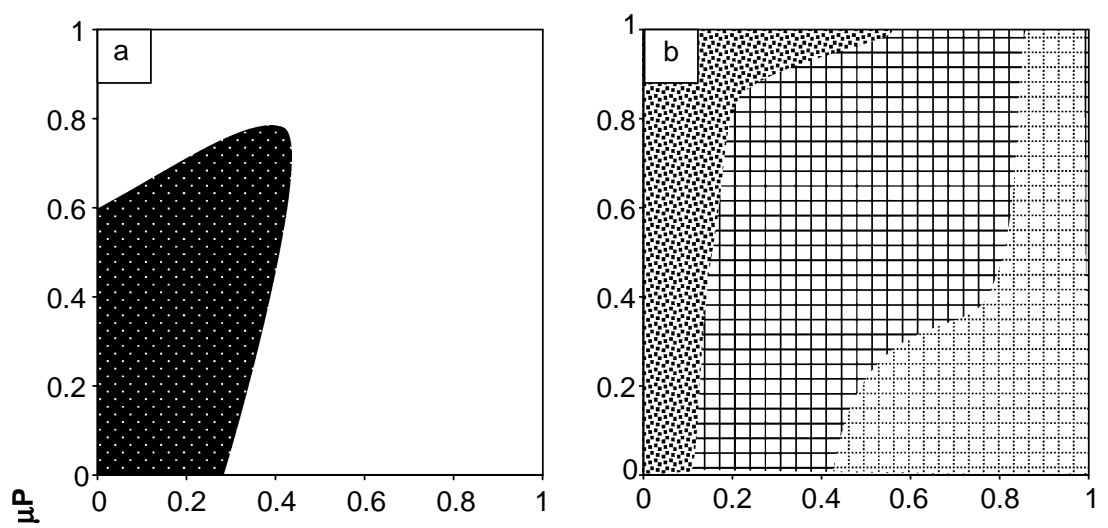

$\square$ No emergence
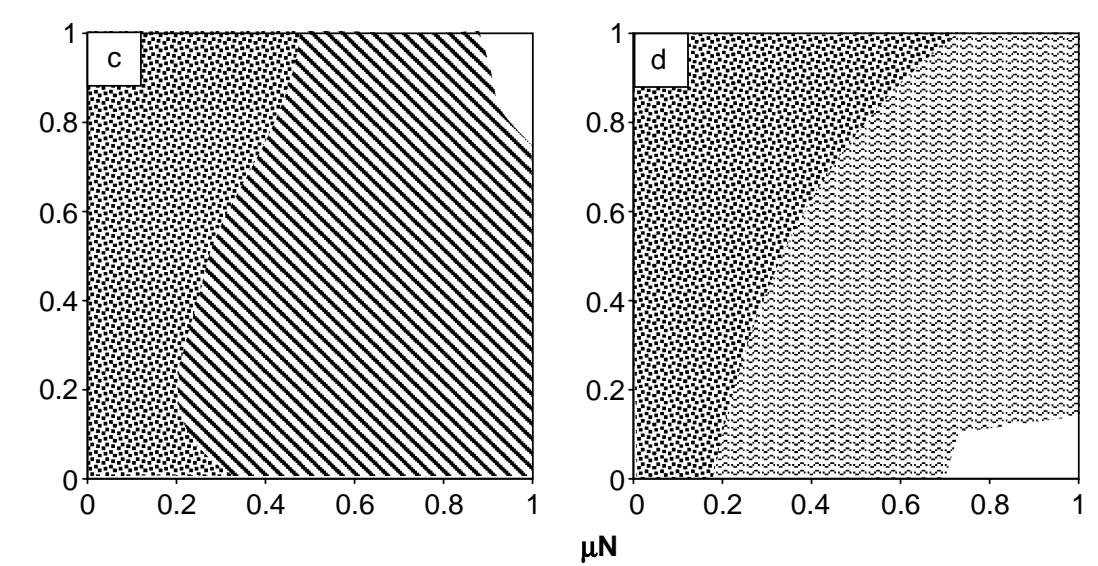
\% spatial chaos \# Spiral frag. or Main.-island i.: Late extinction MN Spiral fragments $\geqslant \notin$ Spirals

Fig. 5. Combination of host $\left(\mu_{\mathrm{N}}\right)$ and parasitoid $\left(\mu_{\mathrm{P}}\right)$ dispersal rates leading to the emergence of different patterns in the four landscape paradigms: (a) spotted, (b) archipelago, (c) labyrinth, (d) Swiss-cheese. These charts contain some degree of subjectivity, as transitions between patterns are gradual rather than abrupt; the notional boundary between the patterns can be interpreted as the middle of a transition zone where hybrid dynamics can be seen. The "no emergence" areas are those where population persistence is unlikely (one or both species goes extinct after a few $(<15)$ generations. Close to these areas, the dynamics tends to be very unstable with episodes of very low global density. In the "late extinction" areas, one or both species goes extinct after a long history ( $>100$ generations) tending toward synchronisation of the whole lattice.

waves. We call this the dispersed reseeding persistence mechanism. Alternatively, if the host is highly vagile, the dynamics are synchronised on a longer range. This forces reseeding locations to be far apart. In a homogeneous landscape this is the process that yields true spirals: near the spiral's vortex, the host population, depleted by the parasitoid wave, is reseeded and can grow into a new wave, generating a persistent, directional (a given spiral never reverses its rotation sense) and symmetrical pattern. We call this the focal reseeding persistence mechanism.

Heterogeneous landscape geometry interferes with these reseeding processes. Dispersed reseeding is quite robust to habitat fragmentation since it is a short-range

Table 2. Two types of qualitative information are sufficient to grossly predict the emergence of each pattern: landscape geometry (rows) and host dispersal rate $(+=$ low,$++=$ medium,$+++=$ high dispersal rate).

\begin{tabular}{|c|c|c|c|c|c|}
\hline Patterns Geometries & Chaos & Meta-population & Mainland-island & Spiral fragments & Spirals \\
\hline Swiss cheese & + & & & \multirow{4}{*}{+++} & \multirow[t]{4}{*}{+++} \\
\hline Labyrinth & + & & & & \\
\hline Archipelago & + & & ++ & & \\
\hline Spotted & \multicolumn{2}{|r|}{+} & & & \\
\hline Persistence mechanism & \multicolumn{2}{|c|}{ Dispersed reseeding } & Host-bridge & \multicolumn{2}{|c|}{ Focal reseeding } \\
\hline
\end{tabular}


mechanism. It occurs with all geometries except the spotted landscape, and generates the spatial chaos pattern. With the spotted landscape, reseeding is constrained to take place in small and isolated patches; the ensuing spatial chaos pattern occurs in the matrix and its only conspicuous effect is to fill or empty the patches pseudo-randomly. Therefore, this pattern is a special case of the spatial chaos pattern with many features hidden in the matrix.

By contrast, the focal reseeding is much more sensitive to landscape geometry. We have seen that full-developed spiral - the spiral pattern - can occur only in Swiss-cheese geometry. In a fragmented landscape like the labyrinth geometry, the spiral's symmetry is broken, but the reseeding occurs by hosts reinvading the depleted patch from a yet untouched one; the process is still directional as the spiral fragments travel a given patch following always the same direction.

For this reseeding to be possible, the patches must be close enough. When they are too far apart, i.e. in the archipelago geometry, the persistence is not possible at high host dispersal rate; however, when it is medium, a hybrid mechanism occurs: dispersed reseeding maintains the biggest patch (the mainland), which in turn reseeds periodically the smallest ones by means of the host-bridge effect.

\section{Discussion}

The simulation results allowed us to distinguish five important patterns. How are they related to previous theoretical and field studies?

The spatial chaos pattern describes how a spatial aggregation may arise even if the landscape structure is mostly homogeneous. The chaotic nature of the wave propagation makes this an unpredictable process and, in the field, it will be difficult to distinguish it from other effects inducing individual aggregation. Moreover, as spatial heterogeneity is a scale-dependent concept, some systems described as metapopulations at some scale could actually be driven by chaotic waves at a larger and thus more homogeneous - scale.

The spiral pattern, although described in many previous theoretical studies (Perry 1995, Rohani et al. 1997, Gurney et al. 1998, Allen et al. 2001) has so far never been observed in real landscapes, although it has been shown to resist robustly a high level of stochasticity (Ruxton and Rohani 1996). Our results show that spiral patterns are easily destroyed by spatial heterogeneity (see also Hassell et al. 1993), in particular when spatial autocorrelation increases landscape irregularity at the scale at which the spirals appear. As the size of these patterns is directly related to the dispersal range of the focal species, it could well be that no real landscape can offer a space large and homogeneous enough to house them. In our simulations, fully developed spirals are rather uncommon since they can only appear when the patches are large and homogeneous enough.

The metapopulation pattern has been the subject of several previous studies (Hanski and Gaggiotti 2004), although it is still unclear how many populations really belong to the classical case as defined by Hanski (1997) rather than to the, apparently more common, mainland-island (or source-sink) one. Most studies focus on a single species without modelling explicitly the causes of local extinctions (but see Holyoak and Lawler 1996, Lei and Hanski 1998, Johst and Schops 2003). Recolonisation of empty patches is a central theme of metapopulation models but, as most of them are built on the base of a snapshot of the patch-occupancy situation, it is difficult to assess if these recolonisations are purely random (as assumed by metapopulation theory) or follow some spatio-temporal pattern. Our simulations show two different situations that could be perceived as metapopulation dynamics if observed within a small time-window: the first is the one we called the metapopulation pattern, where local patches are small, close to each other and whose truly random extinction/recolonisation sequence is generated by an underlying spatial-chaos process; this pattern is uncommon in our simulations since it only emerges in very scarce landscapes and for low host dispersal rates. The second is the spiral-fragment pattern where the patches are larger (as related to dispersal range), further apart and where extinction/recolonisation are driven by the propagation of a wave of parasitoids; this pattern is much more conspicuous for the parasitoid than for the host. Discriminating between these two patterns would need a time-series analysis: whilst the metapopulation pattern shows pseudo-random recolonisation/ extinctions, the spiral fragment pattern predicts them to be regularly recurrent.

The spiral-fragment pattern is actually one of the commonest dynamic patterns emerging from our simulations. Apart from the metapopulations, which one saw it could be confounded with, this pattern is also well supported by field studies. Recurrent, directional, travelling waves are a common phenomenon (Okubo 1980, Ranta et al. 1997, Kaitala and Ranta 1998, Haydon and Greenwood 2000, Grenfell et al. 2001, Murdoch and Briggs 2002). In particular, Bjornstad et al.'s (2002) detailed analysis of the larch budmoth Zeiraphera diniana outbreaks across the Alps shows a pattern strikingly similar to the spiralfragment pattern: directional waves travelling across an elongated patch (the Alps), originating recurrently from its western end.

Finally, the mainland-island pattern, which is a common outcome of our simulations, is too quite often found in real populations (Amezcua and Holyoak 2000). A prediction of the host-bridge process that 
drives this pattern is that, the further an island is from the mainland, the more a prey is released from its predator and, therefore, the larger its cycle amplitude and period. This prediction is well supported by field studies on insect prey-predator systems (Kareiva 1987, 1990, Kruess and Tscharntke 1994).

The patterns exhibited by our simulations seem therefore to correspond to an ecological reality. However, linking theoretical spatial predictions to field observations is always flawed as this consists basically in fitting the predicted results of a process to a detected pattern. Most often, long time-series of spatiallyreferenced data are not available and we are left with snapshots of what is most probably a transient situation. We have seen that in some cases this would not be sufficient to discriminate between two patterns. The spatial extent of the study has also some importance. For instance, in the case of the mainland-island pattern, a study focused on the mainland would conclude at a spatial-chaos pattern while the same study conducted on the islands would find regular outbreaks separated by long time interval. However, there are means to get around these difficulties. When spatially-explicit data are unavailable, it is still possible to determine which pattern it is linked with, provided a sufficiently long time-series on a sufficiently large spatial extent. The host/parasitoid density ratio linked to cycle characteristics will make it possible to select the most probable pattern. Murdoch et al. (2002) claimed that global time-series spectrum analysis was conveying information about ecological relationship at the community scale; here we argue that such analyses can also provide clues about spatial ecology.

\section{Implications for conservation}

Spatial ecology has previously identified a variety of responses of populations to habitat loss and fragmentation. For example, Hanski (1997) distinguishes the "classical metapopulation" (sensu Levins 1969), mainland-island, ephemeral aggregation of individuals, isolated populations and synchronised local populations. These different dynamical scenarios may need different conservation policies and have therefore given rise to distinct theoretical frameworks (Hanski 1997). An interesting outcome of our model is that it can reproduce all these situations - as well as hybrids by varying essentially only three parameters, namely proportion of suitable habitat, spatial autocorrelation (related to fragmentation), and host dispersal rate. Identifying which processes are governing a particular population is one of the first and most important questions a conservation biologist has to answer when tackling a new problem. Therefore, being able to delineate from simple landscape and dynamic probes, which is the most appropriate model among several candidates, i.e. which is the expected pattern, would be a powerful tool for both basic ecology and management.

While confirming that habitat loss and fragmentation are indeed important factors for population dynamics, our study highlights the importance of dispersal rates and interspecies relationships (confirming previous work, Darwen and Green 1996, Bascompte and Solé 1998), since they may determine which pattern is actually occurring in the system. Being based on the NB model, our findings are particularly relevant for systems where consumer and resource are strongly coupled and prone to cycles, whilst systems with generalist consumers are more likely to exhibit single-species-like dynamics (Murdoch et al. 2002). Furthermore, depending on the pattern and whether we are primarily interested in the host or the parasitoid, habitat loss, fragmentation or dispersal may prove to be the crucial parameter, needing either to be carefully monitored or controlled through management. An example of this is the management of predator populations. The habitat suitability map of its main prey is often taken as a predictor of the predator's distribution (Boyce and Mcdonald 1999, Cramer and Portier 2001, Hirzel and Arlettaz 2003, Hirzel et al. 2004). However, as exemplified by the labyrinth geometry, prey spatial distribution may be of limited interest if not considered together with its dispersal behaviour. Biological pest control is another domain that could benefit from this approach. Spatial processes have long been thought important in some pest control situations (Settle et al. 1996). Our model predicts, for example, that field geometry is a dominant factor controlling outbreaks and suggests landscape designs that may help achieve this goal.

\section{Contributions to ecological theory}

The present model provides novel insight in two domains of theoretical ecology. First, it is one further contribution to the long history of NB model stability analysis. Including space, implicitly or explicitly, was known to have a stabilising effect on its dynamics. However, spatially homogeneous systems were producing symmetrical spatial patterns, like spirals or circles, which have never been observed in the field. Our work shows that spatial heterogeneity breaks up these unrealistic symmetrical patterns in most cases, even in absence of stochasticity. These patterns are replaced by a palette of realistic behaviours that can be related to field observations: spatial chaos, spiral-fragments (or travelling waves), metapopulation and mainland-island. Cycles in total population numbers can occur, and have periods in the range expected from non-spatial theory 
( $>6$ ), and shown by Murdoch et al. (2002) to be characteristic of many observed consumer-resource interactions.

Second, our model establishes a conceptual bridge between landscape ecology and population ecology, two domains that, although they are closely related, use such different tools that it is difficult to apply one to the other. Our model places a classical and highly studied host-parasitoid model within a heterogeneous landscape defined by common fragmentation and habitat-loss parameters. For the sake of simplicity, we have here restricted our analysis to exemplary and well-defined landscape geometries, but obviously the next step is to study more precisely how the dynamical characteristics of the system are affected along the whole gradient of landscape configurations.

In this paper we have considered environmental heterogeneity as acting on host growth rate. Future studies could address spatial heterogeneity in the parasitoid attack rate - i.e. spatially explicit host refuges - or in the dispersal rates - i.e. dispersal barriers. Another question of interest would be to study how our results can be generalised to different interaction types: e.g. competitors and food webs (Arditi et al. 2005).

Acknowledgements - We wish to thank E. Pachepsky, M. Fujiwara and N. Perrin for their keen comments at various stages of this work. We thank the Univ. of California at Santa Barbara and its Ecology, Evolution and Marine Biology department for providing the research facilities. We also thank Judit Szabo whose comments helped to improve the manuscript. This work was funded by the Swiss National Science Foundation, grant no. 3100A0-112511 to A.H. Hirzel, US National Science Foundation, grant no. 0108450 to R. M. Nisbet and no. DEB 0089515 to W. W. Murdoch.

\section{References}

Allen, J. C. et al. 2001. Spatially explicit ecological models: a spatial convolution approach. - Chaos Solitons Fractals 12: 333-347.

Amezcua, A. B. and Holyoak, M. 2000. Empirical evidence for predator-prey source-sink dynamics. - Ecology 81: 3087-3098.

Andren, H. 1999. Habitat fragmentation, the random sample hypothesis and critical thresholds. - Oikos 84: 306-308.

Arditi, R. et al. 2005. Rheagogies: modelling non-trophic effects in food webs. - Ecol. Complexity 2: 249-258.

Bascompte, J. and Solé, R. V. 1998. Effects of habitat destruction in a prey-predator metapopulation model. - J. Theor. Biol. 195: 383-393.

Bjornstad, O. N. et al. 2002. Waves of larch budmoth outbreaks in the European Alps. - Science 298: 10201023.

Boyce, M. S. and Mcdonald, L. L. 1999. Relating populations to habitats using resource selection functions. - Trends Ecol. Evol. 14: 268-272.
Comins, H. N. et al. 1992. The spatial dynamics of host parasitoid systems. - J. Anim. Ecol. 61: 735-748.

Cramer, P. C. and Portier, K. M. 2001. Modeling Florida panther movements in response to human attributes of the landscape and ecological settings. - Ecol. Modell. 140: 51-80.

Darwen, P. J. and Green, D. G. 1996. Viability of populations in a landscape. - Ecol. Modell. 85: 165171.

Dussert, C. et al. 1989. Minimal spanning tree approach to percolation and conductivity threshold. - Phys. Lett. A 139: $35-38$

Gomez-Hernandez, J. J. and Srivastava, R. M. 1990. ISIM3D: an ANSI-C three-dimensional multiple indicator conditional simulation program. - Comput. Geosci. 16: 395-440.

Goovaerts, P. 1998. Impact of the simulation algorithm, magnitude of ergodic fluctuations and number of realizations on the spaces of uncertainty of flow predictions. - Stanford Univ.

Grenfell, B. T. et al. 2001. Travelling waves and spatial hierarchies in measles epidemics. - Nature 414: 716723.

Gurney, W. S. C. and Nisbet, R. M. 1978. Predator-prey fluctuations in patchy environments. - J. Anim. Ecol. 47: $85-102$.

Gurney, W. S. C. and Nisbet, R. M. 1998. Ecological dynamics. - Oxford Univ. Press.

Gurney, W. S. C. et al. 1998. Circle and spirals: population persistence in a spatially explicit predator-prey model. - Ecology 79: 2516-2530.

Hanski, I. 1997. Metapopulation dynamics: from concepts and observations to predictive models. - In: Hanski, I. and Gilpin, M. E. (eds), Metapopulation biology: ecology, genetics, and evolution. Academic Press, pp. 69-91.

Hanski, I. and Gaggiotti, O. E. (eds) 2004. Ecology, genetics and evolution of metapopulations. - Elsevier.

Hargrove, W. W. et al. 2002. A fractal landscape realizer for generating synthetic maps. Conserv. Ecol. [online], p. 2.

Hassell, M. P. et al. 1993. Effects of global change on the dynamics of insect host-parasitoid interactions. - In: Kareiva, P. and Kingsolver, J. (eds), Biotic interactions and global change. Sinauer, pp. 402-423.

Haydon, D. T. and Greenwood, P. E. 2000. Spatial coupling in cyclic population dynamics: models and data. - Theor. Popul. Biol. 58: 239-254.

Hiebeler, D. 2000. Populations on fragmented landscapes with spatially structured heterogeneities: landscape generation and local dispersal. - Ecology 81: 1629-1641.

Hill, M. F. and Caswell, H. 1999. Habitat fragmentation and extinction thresholds on fractal landscapes. - Ecol. Lett. 2: $121-127$

Hirzel, A. H. 2001. When GIS come to life. Linking landscape- and population ecology for large population management modelling: the case of ibex (Capra ibex) in Switzerland. Inst. of Ecol., Lab. for Conserv. Biol. - Univ. of Lausanne, p. 108.

Hirzel, A. H. and Arlettaz, R. 2003. Modelling habitat suitability for complex species distributions by the 
environmental-distance geometric mean. - Environ. Manage. 32: 614-623.

Hirzel, A. H. et al. 2004. Ecological requirements of a reintroduced species, with implications for release policy: the bearded vulture recolonizing the Alps. - J. Appl. Ecol. 41: $1103-1116$.

Holyoak, M. and Lawler, S. P. 1996. The role of dispersal in predator-prey metapopulation dynamics. - J. Anim. Ecol. 65: $640-652$.

Johst, K. and Schops, K. 2003. Persistence and conservation of a consumer-resource metapopulation with local overexploitation of resources. - Biol. Conserv. 109: 57-65.

Kaitala, V. and Ranta, E. 1998. Travelling wave dynamics and self-organization in a spatio- temporally structured population. - Ecol. Lett. 1: 186-192.

Kareiva, P. 1987. Habitat fragmentation and the stability of predator prey interactions. - Nature 326: 388-390.

Kareiva, P. 1990. Population-dynamics in spatially complex environments-theory and data. - Philos. Trans. R. Soc. Lond. B 330: 175-190.

Kruess, A. and Tscharntke, T. 1994. Habitat fragmentation, species loss, and biological-control. - Science 264: 15811584 .

Lei, G. C. and Hanski, I. 1998. Spatial dynamics of two competing specialist parasitoids in a host metapopulation. - J. Anim. Ecol. 67: 422-433.

Levins, R. 1969. Some demographic and genetic consequences of environmental heterogeneity for biological control. - Bull. Entomol. Soc. Am. 15: 237-240.

Maynard Smith, J. 1974. Models in ecology. - Cambridge Univ. Press.

Mcgarigal, K. et al. 2002. FRAGSTATS: spatial pattern analysis program for categorical maps. University of Massachussetts. URL: http://www.umass.edu/landeco/research/fragstats/fragstats.html

Murdoch, W. W. and Briggs, C. J. 2002. Spatial dynamics of measles epidemics. - Trends Ecol. Evol. 17: 399-401.

Murdoch, W. W. et al. 1992. Aggregation and stability in metapopulation models. - Am. Nat. 140: 41-58.

Murdoch, W. W. et al. 2002. Single-species models for manyspecies food webs. - Nature 417: 541-543.

Murdoch, W. W. et al. 2003. Consumer-resource dynamics. - Princeton Univ. Press.

Nicholson, A. J. and Bailey, V. A. 1935. The balance of animal populations. Part 1. - Proc. Zool. Soc. Lond. 3: $551-598$.

Okubo, A. 1980. Diffusion and ecological problems: mathematical models. - Springer.

Perry, D. A. 1995. Self-organizing systems across scales. - Trends Ecol. Evol. 10: 241-244.

Press, W. H. et al. 1989. Numerical recipes in Pascal: the art of scientific computing. - Cambridge Univ. Press.

Pulliam, H. R. 1988. Sources, sinks, and population regulation. - Am. Nat. 132: 652-661.

Ranta, E. et al. 1997. Dynamics of Canadian lynx populations in space and time. - Ecography 20: 454-460.

Rohani, P. et al. 1997. Spatial self-organization in ecology: pretty patterns or robust reality? - Trends Ecol. Evol. 12: $70-74$.

Ruxton, G. D. and Rohani, P. 1996. The consequences of stochasticity for self-organized spatial dynamics, persistence and coexistence in spatially extended hostparasitoid communities. - Proc. R. Soc. Lond. B 263: 625-631.

Settle, W. H. et al. 1996. Managing tropical rice pests through conservation of generalist natural enemies and alternative prey. - Ecology 77: 1975-1988.

Sherratt, J. A. 2001. Periodic travelling waves in cyclic predator-prey systems. - Ecol. Lett. 4: 30-37.

Solé, R. V. et al. 1992. Spiral waves, chaos and multiple attractors in lattice models of interacting populations. - Phys. Lett. A 166: 123-128.

Thomas, C. D. and Kunin, W. E. 1999. The spatial structure of populations. - J. Anim. Ecol. 68: 647-657.

Tischendorf, L. 2001. Can landscape indices predict ecological processes consistently? - Landscape Ecol. 16: 235254.

With, K. A. 1997. The application of neutral landscape models in conservation biology. - Conserv. Biol. 11: 1069-1080.

With, K. A. and King, A. W. 1997. The use and misuse of neutral landscape models in ecology. - Oikos 79: 219229.

\section{Appendix 1. Diffusion effect}

The diffusion effect may lead a patch to extinction providing that it propagates more dispersers it can compensate for by reproduction and immigration. This effect - which is related to the critical patch size theory - depends primarily on the dispersal rate $\mu_{N}$, the number of adjacent habitat hexes $\mathrm{k}^{\prime}$ and the growth rate $\lambda$, and more marginally on the distance to other sources of host dispersers. Neglecting predation and immigration, equation 1a shows that, for a hex not to go extinct, the following relation must be true:

$\mu_{\mathrm{N}} \leq \frac{\lambda_{\mathrm{H}}-1}{\lambda_{\mathrm{H}}\left(1-\frac{\mathrm{k}^{\prime}}{\mathrm{k}}\right)}$

With the parameter values used throughout this paper $\left(\lambda_{\mathrm{H}}=2, \mathrm{k}=6\right)$, this entails that any hex with three or more neighbours will be viable for any value of $\mu_{N}$, and, conversely, if $\mu_{\mathrm{N}} \leq 0.5$, even a single isolated hex is viable.

\section{Appendix 2. Host-parasitoid dynamics with constant inflow}

We consider here a version of the NB model where the focal system is an island experiencing a constant inflow of hosts $I_{N}$ and parasitoids $I_{P}$ from a close enough mainland so that dispersers cross the gap in one generation:

$\mathrm{N}_{\mathrm{t}+1}=\lambda \mathrm{N}_{\mathrm{t}} \exp \left\{-\mathrm{aP}_{\mathrm{t}}\right\}+\mathrm{I}_{\mathrm{N}}$ 
$\mathrm{P}_{\mathrm{t}+1}=\mathrm{N}_{\mathrm{t}} \mathrm{c}\left(1-\exp \left\{-\mathrm{aP}_{\mathrm{t}}\right\}\right)+\mathrm{I}_{\mathrm{P}}$

Many dynamical properties of this system can be demonstrated by standard approaches involving the computation of equilibria and by the study of small perturbations from an equilibrium state (Gurney and Nisbet 1998, chapter 3, Murdoch et al. 2003, chapter 4). These analyses show that the system is persistent provided both immigration rates are strictly positive $\left(\mathrm{I}_{\mathrm{N}}\right.$ $>0$ and $\left.I_{P}>0\right)$. If both of them are small there are typically persistent limit cycles, while if either is sufficiently large there is a stable equilibrium. With $\lambda=2$ and $\mathrm{a}=\mathrm{c}=1$, we find empirically that the period of the cycles linearly decreases with $\log \left(\mathrm{I}_{\mathrm{N}}\right)$. After a parasitoid-driven extinction, the host can always recover thanks to immigration, but the smaller the inflow, the longer the recovery time. However, the host cannot recover if the inflow is insufficient to keep the population above the extinction threshold; for a threshold of $10^{-10}$, the maximum period is around 36 generations. Parasitoid inflow has little effect on oscillation period, however the cycle amplitude decreases non-linearly as parasitoid amplitude increases. On or near the stability boundary, the period does not drop much below 6 when $\lambda<10$ - a property similar to that noted for a wide range of consumer-resource systems by Murdoch et al. (2002).

\section{Appendix 3. Mainland-island dispersal: the host-bridge effect}

Let us consider a mainland assumed to be large enough to convey constant populations of hosts and parasitoids, separated from an island by a space of unsuitable habitat, which takes $\mathrm{D}$ generations to be crossed. This one-dimensional system can be modelled in the framework of Eq. 1 and 2, with a neighbourhood size $\mathrm{k}=2$ (dispersers can only move islandward or mainlandward). The system is composed of $\mathrm{D}+2$ cells with reflexive boundary conditions. At one end lies the mainland with constant densities $\mathrm{N}_{0}=\mathrm{P}_{0}=1$ and therefore constant numbers of dispersers. At the other end is the suitable island with a growth rate $\lambda=\lambda_{\mathrm{H}}=2$. In between are the unsuitable cells with $\lambda=\lambda_{M}=0.01$; these cells can therefore not sustain populations without immigrants from either the island or the mainland.

In the absence of any island, the population densities in the unsuitable cells quickly achieve death-immigration equilibrium. A numerical model showed that the logarithm of host equilibrium density $\mathrm{N}_{\mathrm{i}}^{\prime}$ decreases linearly with distance $\mathrm{i}$ from mainland, whilst the logarithm of the parasitoid equilibrium density $\mathrm{P}_{\mathrm{i}}^{\prime}$ decreases quadratically. This can be confirmed mathematically as follows:
In an one-dimensional system, dispersal Eq. 1 become:

$\mathrm{N}_{\mathrm{i}, \mathrm{t}}^{\prime}=\mathrm{N}_{\mathrm{i}, \mathrm{t}}-\mu_{\mathrm{N}} \mathrm{N}_{\mathrm{i}, \mathrm{t}}+\frac{1}{2} \mu_{\mathrm{N}} \mathrm{N}_{\mathrm{i}-1, \mathrm{t}}+\frac{1}{2} \mu_{\mathrm{N}} \mathrm{N}_{\mathrm{i}+1, \mathrm{t}}$

$\mathrm{P}_{\mathrm{i}, \mathrm{t}}^{\prime}=\mathrm{P}_{\mathrm{i}, \mathrm{t}}-\mu_{\mathrm{N}} \mathrm{P}_{\mathrm{i}, \mathrm{t}}+\frac{1}{2} \mu_{\mathrm{N}} \mathrm{P}_{\mathrm{i}-1, \mathrm{t}}+\frac{1}{2} \mu_{\mathrm{N}} \mathrm{P}_{\mathrm{i}+1, \mathrm{t}}$

The host-parasitoid step then takes the form

$\mathrm{N}_{\mathrm{i}, \mathrm{t}+1}=\lambda_{\mathrm{M}} \mathrm{N}_{\mathrm{i}, \mathrm{t}}^{\prime} \exp \left\{-\mathrm{aP}_{\mathrm{i}, \mathrm{t}}^{\prime}\right\}$

$\mathrm{P}_{\mathrm{i}, \mathrm{t}+1}=\mathrm{N}_{\mathrm{i}, \mathrm{t}}^{\prime}\left(1-\exp \left\{-\mathrm{aP}_{\mathrm{i}, \mathrm{t}}^{\prime}\right\}\right)$

The approximation is based on the assumptions that matrix growth rate is low and the densities decrease rapidly from one cell to the next, i.e. $\lambda_{\mathrm{M}} \ll 1 ; \mathrm{N}_{\mathrm{i}} \ll$ $\mathrm{N}_{\mathrm{i}-1} ; \mathrm{P}_{\mathrm{i}} \ll \mathrm{P}_{\mathrm{i}-1}$. Furthermore, we are only interested in equilibrium solutions, so we drop the subscript t. With these approximations, Eq. 5a and $5 \mathrm{~b}$ can be simplified to the form:

$\mathrm{N}_{\mathrm{i}}^{\prime}=\frac{1}{2} \mu_{\mathrm{N}} \mathrm{N}_{\mathrm{i}-1}$

$\mathrm{P}_{\mathrm{i}, \mathrm{t}}^{\prime}=\frac{1}{2} \mu_{\mathrm{N}} \mathrm{P}_{\mathrm{i}-1}$

With the same assumptions as above, Eq. 2a then becomes:

$\mathrm{N}_{\mathrm{i}} \approx \frac{1}{2} \lambda_{\mathrm{M}} \mu_{\mathrm{N}} \cdot \mathrm{N}_{\mathrm{i}-1}$

This approximation is likely to be excellent for all but the smallest values of $i$ (and is of course exact when there are no parasitoids. Thus we expect that asymptotically the host density decreases geometrically with distance into the matrix and we can conjecture that to a good level of approximation

$\log \left(\mathrm{N}_{\mathrm{i}}^{\prime}\right)=\log (\mathrm{C})+\log (\mathrm{A}) \mathrm{i}$

with $\mathrm{A}=\frac{1}{2} \lambda_{\mathrm{M}} \mu_{\mathrm{N}}$ and $\mathrm{C}$ a positive constant depending on $\mathrm{N}_{0}$ and $\mathrm{P}_{0}$. Similarly, we find that, for the parasitoid, we have:

$\mathrm{Pi}=\frac{1}{4} \mathrm{a} \mu_{\mathrm{P}} \mu_{\mathrm{N}} \mathrm{N}_{\mathrm{i}-1} \mathrm{P}_{\mathrm{i}-1}=\mathrm{BCA}^{\mathrm{i}} \mathrm{P}_{\mathrm{i}-1}$

with $\mathrm{B}=\frac{1}{4} \mathrm{a} \mu_{\mathrm{P}} \mu_{\mathrm{N}}$

Thus

$\log \left(\mathrm{P}_{\mathrm{i}}\right)=\log (\mathrm{BC})+\mathrm{i} \log (\mathrm{A})+\log \left(\mathrm{P}_{\mathrm{i}-1}\right)$

which, if exactly true for all $\mathrm{i}$ would imply that 


$$
\begin{aligned}
\log \left(P_{i}^{\prime}\right)= & i \log (B C)+\log (A) \sum_{j=0}^{i} j=i \log (B C) \\
& +\log (A) \frac{1}{2} i(i+1)+\log \left(P_{0}\right)
\end{aligned}
$$

Thus $\log \left(\mathrm{P}_{\mathrm{i}}^{\prime}\right)$ varies quadratically with i, i.e.

$$
\log \left(\mathrm{P}_{\mathrm{i}}^{\prime}\right)=\alpha+\beta \mathrm{i}+\gamma \mathrm{i}^{2} \text { with } \gamma=\frac{1}{2} \log \left(\frac{1}{2} \lambda_{\mathrm{M}} \mu_{\mathrm{N}}\right)
$$

This means that, whenever an extinction threshold prevents a population to get infinitesimally small, host dispersers can reach further away from the mainland than the parasitoids. If the island can initially be reached by the host and not by the parasitoid, the former will grow there exponentially. As the island host density increases, it will become a significant source of dispersers spreading toward the mainland. At some time, which depends on the distance $\mathrm{D}$ between the mainland and the continent, there will be enough host dispersers in the interpatch space to allow the parasitoid to cross it. This is what we call the "host-bridge effect". The parasitoid invasion of the island drives both populations extinct there, and the process starts again. This generates cyclic dynamics with regular host outbreaks closely followed by short bursts of parasitoids. The cycle period increases linearly with the distance D; the slope of this increase is fairly insensitive to other parameters, except for high host dispersal rates $\mu_{\mathrm{N}}$, which makes the slope steeper.

\section{Appendix 4. Sequential Gaussian algorithm}

The sequential Gaussian simulation algorithm used for spatially correlated stochasticity was derived from Gomez-Hernandez and Srivastava (1990), as well as from Goovaerts (1998) with a few modifications designed to adapt it to torus topology. The principle is to generate for each hex $\mathrm{x}_{\mathrm{i}}$ a spatially correlated and normally distributed (mean $=0, \quad S D=1)$ random value $\mathrm{z}_{\mathrm{i}}$ so that they reproduce a Gaussian correlogram model, i.e.:

$r(d)=r_{\text {max }} e^{-\frac{3 d^{2}}{\alpha^{2}}}$

where $r(d)$ is the spatial correlation between two hexes separated by a distance $d, r_{\max }$ is the maximal correlation for $d>0$ and $\alpha$ is the range, i.e. the distance at which $\mathrm{r}(\alpha)=0.05$. The distance $\mathrm{d}$ between two hexes is measured according to the torus topology. The landscape generation did not differ whether absorbing or reflecting boundary conditions were used.
Suppose that we know the $\mathrm{z}$ value at a set of hexes $\left\{\mathrm{x}_{1}, \mathrm{x}_{2}, \ldots, \mathrm{x}_{\mathrm{k}}\right\}$ and we want to draw a random $\mathrm{z}$ value for a cell $x_{k+1}$, so that, together, they reproduce the correlogram model. Geostatistics have developed a family of least-square regression algorithms called "kriging" that answer this question. Conceptually, "simple kriging" is a locally-weighted linear regression. It expresses the estimated value $\mathrm{z}^{*}\left(\mathrm{x}_{\mathrm{k}+1}\right)$ as a linear combination of the $\mathrm{z}\left(\mathrm{x}_{\mathrm{i}}\right)$ :

$\mathrm{Z}^{*}\left(\mathrm{X}_{\mathrm{k}+1}\right)=\sum_{\mathrm{i}=1}^{\mathrm{k}} \mathrm{W}_{\mathrm{i}} \mathrm{Z}\left(\mathrm{X}_{\mathrm{i}}\right)$

The weights $\mathrm{w}_{\mathrm{i}}$ are set according to the relative spatial position of the $\mathrm{x}_{\mathrm{i}}$ and to the correlogram model, so as to minimise the error variance. This is achieved by solving a system of linear equations:

$$
\left\{\begin{array}{c}
\mathrm{r}\left(\mathrm{d}_{1, \mathrm{k}+1}\right)=\sum_{\mathrm{i}=1}^{\mathrm{k}} \mathrm{w}_{\mathrm{i}} \mathrm{r}\left(\mathrm{d}_{1, \mathrm{i}}\right) \\
\mathrm{r}\left(\mathrm{d}_{2, \mathrm{k}+1}\right)=\sum_{\mathrm{i}=1}^{\mathrm{k}} \mathrm{w}_{\mathrm{i}} \mathrm{r}\left(\mathrm{d}_{2, \mathrm{i}}\right) \\
\vdots \\
\mathrm{r}\left(\mathrm{d}_{\mathrm{k}, \mathrm{k}+1}\right)=\sum_{\mathrm{i}=1}^{\mathrm{k}} \mathrm{w}_{\mathrm{i}} \mathrm{r}\left(\mathrm{d}_{\mathrm{k}, \mathrm{i}}\right)
\end{array}\right.
$$

where $d_{i, i}$ is the distance between cells $x_{i}$ and $x_{j}$.

Furthermore, kriging theory shows that the variance of $\mathrm{z}^{*}$ is given by:

$\sigma^{2}\left(\mathrm{x}_{\mathrm{k}+1}\right)=1-\sum_{\mathrm{i}=1}^{\mathrm{k}} \mathrm{w}_{\mathrm{i}} \mathrm{r}\left(\mathrm{d}_{\mathrm{k}+1, \mathrm{i}}\right)$

with the constraint that, for $\sigma^{2}$ to be positive, the correlogram model $\mathrm{r}$ must produce a positive-definite correlation matrix.

From here, the sequential Gaussian algorithm proceeds as follows:

1. Define a path $\left\{\mathrm{x}_{1}, \mathrm{x}_{2}, \ldots, \mathrm{x}_{\mathrm{N}}\right\}$ visiting each hex once and only once, in random order.

2. For each hex $x_{i}$ taken sequentially along the path do:

3. Using Eq. 15, 16 and 17, compute its expected mean $\mathrm{z}^{*}\left(\mathrm{x}_{\mathrm{i}}\right)$ and $\mathrm{SD} \sigma^{2}\left(\mathrm{x}_{\mathrm{i}}\right)$, on the base of the hexes already generated $\left\{\mathrm{x}_{1}, \mathrm{x}_{2}, \ldots, \mathrm{x}_{\mathrm{i}-1}\right\}$.

4. Draw a random value from a Gaussian distribution with mean $\mathrm{z}^{*}\left(\mathrm{x}_{\mathrm{i}}\right)$ and $\mathrm{SD} \sigma^{2}\left(\mathrm{x}_{\mathrm{i}}\right)$, and assign it to $\mathrm{z}\left(\mathrm{x}_{\mathrm{i}}\right)$.

5. Proceed to the next hex along the path and repeat steps 3 and 4 until all hexes have been generated.

This algorithm is implemented into a dedicated program (GENESIS, Hirzel, unpubl.), available upon request from $\mathrm{AHH}$. 